\title{
Lane Formation Dynamics of Oppositely Self-Driven Binary Particles: Effects of Density and Finite System Size
}

\author{
Kosuke Ikeda ${ }^{1}$ and Kang $\mathrm{Kim}^{2}$, \\ ${ }^{1}$ Graduate School of Science and Technology, Niigata University, Niigata 950-2181, Japan \\ ${ }^{2}$ Division of Chemical Engineering, Graduate School of Engineering Science, \\ Osaka University, Toyonaka, Osaka 560-8531, Japan
}

\begin{abstract}
We examined the lane formation dynamics of oppositely self-driven binary particles by molecular dynamics simulations of a two-dimensional system. Our study comprehensively revealed the effects of the density and system size on the lane formation. The phase diagram distinguishing the no-lane and lane states was systematically determined for various combinations of the anisotropic friction coefficient and the desired velocity. A peculiar clustered structure was observed when the lane was destroyed by considerably increasing the desired velocity. A strong system size effect was demonstrated by the relationship between the temporal and spatial scales of the lane structure. This system size effect can be attributed to an analogy with the driven lattice gas. The transport efficiency was characterized from the scaling relation in terms of the degree of lane formation and the interface thickness between different lanes.
\end{abstract}

\section{INTRODUCTION}

A recent challenge in research on active matter systems has been understanding the basic principles of various selforganizations observed in the collections of self-propelled or self-driven elements [1-4]. Representative examples include schools of fish, flocks of birds, swarms of animals, and crowds of humans. In these systems, all elements exhibit macroscopic and synchronized collective behavior in the same direction despite microscopic repulsive interactions. The self-driven force can lead to the spontaneous symmetry breaking of the locomotion, which is significantly different from passive Brownian motion.

Lane formation is a remarkable example of selforganization in many autonomous agent systems, and is associated with pedestrian counterflows [5-7]. Specifically, when two groups of agents are driven and move in opposite directions, the agents of the same species tend to move collectively and eventually form a lane in the driven direction. Such segregation of the different lanes is closely related to how efficiently the pedestrians can flow toward the desired direction in various crowded environments, such as evacuation in the event of a disaster [8, 9].

The social force model was proposed to obtain the underlying mechanism of a pedestrian flow from the microscopic level [10, 11]. In this model, each pedestrian is modeled as a particle with a finite excluded volume that interacts via repulsion to avoid collisions. Additionally, the self-driven force acts on each particle in order to adjust the instantaneous velocity to the preassigned desired direction and magnitude. A large number of simulation studies have been carried out to optimize realistic pedestrian behavior [12-16].

A similar lane-formation phenomenon has also been demonstrated in simulations of binary oppositely charged particles [17-31] as well as experiments [32-35]. The phase diagram of the lane formation has been examined for various combinations of the external force and the density under the

\footnotetext{
*kk@ cheng.es.osaka-u.ac.jp
}

periodic boundary condition [17-21, 31] or with confined geometry [27, 29]. The finite-size effect has also been investigated [25, 27, 29]. In particular, the critical force that generates lane formation exhibits a logarithmic increase with the system size, which implies that global phase segregation cannot occur in a finite amount of time [25]. Recently, the observed system size dependence has been interpreted through an analogy with the driven lattice gas model [30]. That is, the lane formation in the driven system is basically governed by the phase separation. However, global lane formation extending to the system size from the disordered and random configuration would occur with infinite time.

With regard to the oppositely self-driven particles, recent advances have been seen in simulations for the phase diagram of lane formation with square system geometry under the periodic boundary condition [36]. In particular, the anisotropic friction perpendicular to the driven direction has been introduced, which mimics the anisotropic response of pedestrians after collisions. However, the information regarding the density and finite-size effects is still limited, in contrast to the data obtained in the above simulation studies on driven systems under an external field.

In this study, we examined the lane formation dynamics of oppositely self-driven binary particles by simulating Langevin molecular dynamics of a two-dimensional system. In particular, we systematically examined the effects of the density and finite size on lane formation. Various order parameters regarding the lane structure were quantified: the degree of lane formation, velocity fluctuation, transport efficiency, characteristic time scale of lane formation, lane number, lane width, and interface thickness between different lanes. Such comprehensive quantitative analysis allowed us to clarify both the similarity and differences between self-driven and externalfield-driven lane formations.

This paper is organized as follows. In Sect. 2, we introduce the simulation model and the numerical details. In Sect. 3 , we define the various observables to characterize the lane formation. In Sect. 4, we present the numerical calculations. Finally, in Sect. 5 we summarize the results. 


\section{SIMULATION MODEL AND DETAILS}

We simulated the Brownian molecular dynamics for twodimensional equimolar binary mixtures of $N$ particles in a regular square. In the mode, each particle is assigned the variable $s=+$ or - . Half of the particles have $s=+$, and the other half have $s=-$. This variable $s$ characterizes the driven direction (along the $x$-axis) when a self-driven force is applied to the particle, which is explained below. The number density is $\rho=N / L^{2}$, where $L$ is the linear dimension of the system. The periodic boundary condition is used in both the $x$ - and $y$ directions. The equation of motion for the position $\boldsymbol{r}_{i}(t)$ and velocity $\boldsymbol{v}_{i}(t)=d \boldsymbol{r}_{i}(t) / d t=\left(v_{i x}, v_{i y}\right)$ of the $i$-th particle is given by

$$
m \frac{d \boldsymbol{v}_{i}}{d t}=-\nabla_{i} \sum_{j \neq i} \phi\left(r_{i j}\right)-\Gamma \boldsymbol{v}_{i}+\boldsymbol{R}_{i}(t)+\boldsymbol{F}_{i}^{(\mathrm{d})} .
$$

Here, $m$ denotes the particle mass. $\phi\left(r_{i j}\right)$ is the interaction potential, which depends only on the pair separation $r_{i j}=\left|\boldsymbol{r}_{i}-\boldsymbol{r}_{j}\right|$ between particles $i$ and $j$. The purely repulsive soft-core potential $\phi(r)=\epsilon(\sigma / r)^{12}$ is used with the cutoff length $r_{c}=2.5 \sigma$. Here, $\epsilon$ and $\sigma$ represent the magnitude of the potential energy and particle diameter, respectively. In addition, $\boldsymbol{R}_{i}(t)$ stands for the zero mean random force acting on the $i$-th particle. The variance of $\boldsymbol{R}_{i}(t)$ is given by $\left\langle R_{i \alpha}(t) R_{j \beta}\left(t^{\prime}\right)\right\rangle=2 m k_{B} T \Gamma \delta_{i j} \delta_{\alpha \beta} \delta\left(t-t^{\prime}\right)$, which is characterized by the thermal energy $k_{B} T$ and the friction coefficient $\Gamma$ that should be associated with the collisions with the solvent. $\alpha$ and $\beta$ represent the two Cartesian components.

The self-driven force acting on particle $i$ is given by

$$
\boldsymbol{F}_{i}^{(\mathrm{d})}=-\gamma_{x}\left(v_{i x}-s_{i} V_{0}\right) \boldsymbol{e}_{x}-\gamma_{y} v_{i y} \boldsymbol{e}_{y}
$$

Here, the first term denotes Helbing's proposed social force model related to pedestrian phenomena [6]. The $x$ component of the $i$-th particle velocity tends to attain the desired velocity $V_{0}$ in the driven $x$-direction, which is determined by the variable $s_{i}$. The corresponding Péclet number $P e$ is then described by $P e=\gamma_{x} V_{0} \sigma / k_{B} T$. This model includes the relaxation time due to the friction $\gamma_{x}$ in the $x$-direction. For the $y$-direction, on the other hand, the $y$-component of the velocity decays to zero because of the friction $\gamma_{y}$ perpendicular to the driven direction. Here, the friction coefficients $\gamma_{x}$ and $\gamma_{y}$ are chosen independently to imitate the anisotropic effect of the momentum exchange with the substrate [36]. The drift velocity of the single particle in the stationary state is described by $\left\langle v_{\text {st }}^{s}\right\rangle=s \gamma_{x} V_{0} /\left(\gamma_{x}+\Gamma\right)$. If the random force $\boldsymbol{R}_{i}(t)$ and associated friction force $-\Gamma \boldsymbol{v}_{i}$ are not taken into account, our model equation reduces to that used in the previous study [36]. In addition, if the frictions $\gamma_{x}$ and $\gamma_{y}$ become zero, the considered equation of motion reduces to the usual Langevin equation of the many-particle system.

Hereafter, the units of length, temperature, and time are represented as $\sigma, \epsilon / k_{B}$, and $\sqrt{m \sigma^{2} / \epsilon}$, respectively. In all simulations, $m=1, T=1$, and $\gamma_{x}=1$ were fixed values, and a time step of $\Delta t=0.001$ was used. The dependence of the number density $\rho$ on the lane formation for various $V_{0}$ and $\gamma_{y}$ was first intensively investigated at the fixed particle number $N=108$. The examined densities were $\rho=0.2,0.3,0.4$, $0.5,0.6,0.7,0.8,0.9$, and 1.0. Next, we focused on the finitesize effect on lane formation by changing the particle number as $N=108,200,400,1000,4000$, and 10000 at the fixed number density $\rho=0.8$. After equilibration with the thermal energy $k_{B} T=1$ using the Langevin equation, the self-driven force $\boldsymbol{F}^{(\mathrm{d})}$ was applied to the particles. In this study, 10 independent $t=10^{3}(N=108)$ and $t=2 \times 10^{4}(N=200$, $400,1000,4000$, and 10000) trajectories were generated, from which various quantities were calculated.

\section{ORDER PARAMETERS}

To characterize the degree of lane formation, some order parameters were proposed for evaluating the number of particles of the same species along the driven direction. As introduced in Ref. 36, the simulation system can be virtually divided into identical rectangular strips along the driven $x$ direction. The number of strips is given by $n_{\mathrm{div}}=[L / \sigma]+1$, where $[\cdots]$ denotes the Gauss symbol. Thus, the width of each strip along the $y$-direction is described by $\ell_{\text {div }}=L / n_{\text {div }}$, which is closer to the particle diameter $\sigma$. The order parameter for the lane formation in the $k$-th rectangular strip is defined as

$$
\psi_{k}=\frac{n_{k}^{+}-n_{k}^{-}}{n_{k}^{+}+n_{k}^{-}}
$$

where $n_{k}^{ \pm}$represents the number of $s= \pm$ species particles in the $k$-th rectangular strip. This signed variable $\psi_{k}$ is determined by the difference in numbers between the positively and negatively driven particles $n_{k}^{+}-n_{k}^{-}$, which is normalized by the total particle number $n_{k}^{+}+n_{k}^{-}$in the $k$-th strip. Thus, the range of $\psi_{k}$ is from -1 to 1 , and $\psi_{k}$ becomes positive if the $s=+$ particles become dominant in the strip and negative if the $s=-$ particles become dominant. The order parameter $\Psi$ for the total system is then defined by

$$
\Psi=\left\langle\frac{1}{n_{\mathrm{div}}} \sum_{k=1}^{n_{\mathrm{div}}}\left|\psi_{k}\right|\right\rangle,
$$

where $\langle\cdots\rangle$ is the ensemble and time average. The order parameter $\Psi$ begins with around zero at the initial state and finally reaches 1 when the system exhibits the lane formation in the steady state. The former corresponds to the randomly mixed state, whereas the latter corresponds to the lane formation structure by the particles of the same species along the driven direction.

The transport property is depicted by the driven efficiency in the $x$-direction, i.e., the average drift velocity normalized by the single particle $\left\langle v_{x}^{s}\right\rangle$,

$$
\eta=\frac{1}{2}\left(\frac{\left\langle\bar{v}_{x}^{+}\right\rangle}{\left\langle v_{\mathrm{st}}^{+}\right\rangle}+\frac{\left\langle\bar{v}_{x}^{-}\right\rangle}{\left\langle v_{\mathrm{st}}^{-}\right\rangle}\right)
$$

where $\bar{v}_{x}^{ \pm}=(2 / N) \sum_{i=1}^{N / 2} v_{i x}^{ \pm}$is the average magnitude of the particle velocity in each driven direction. The velocity fluctu- 


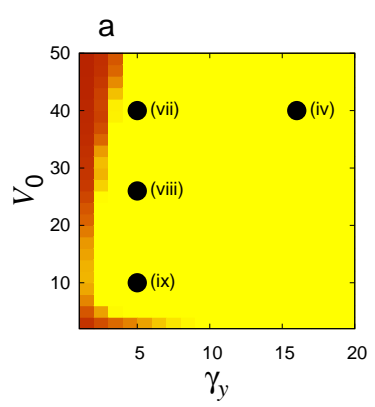

(i)

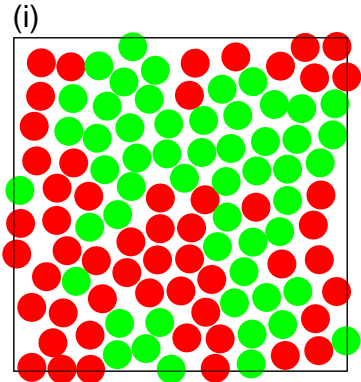

(iv)
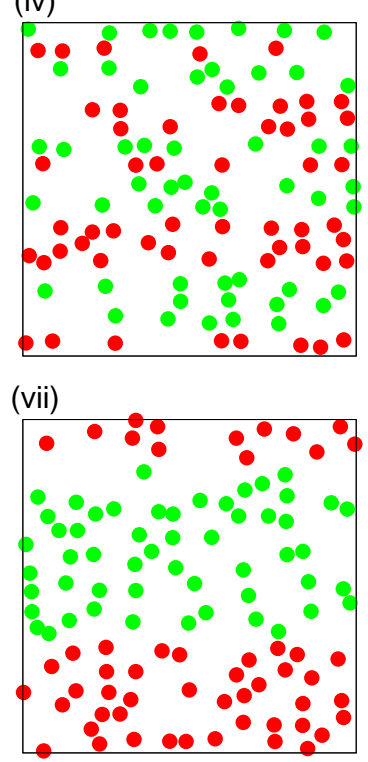

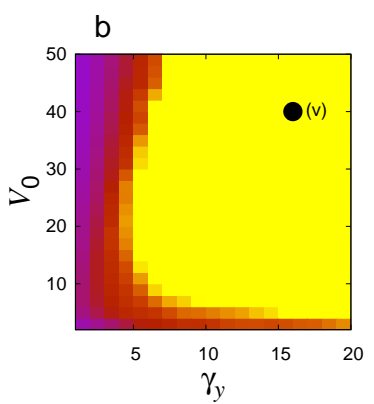

(ii)
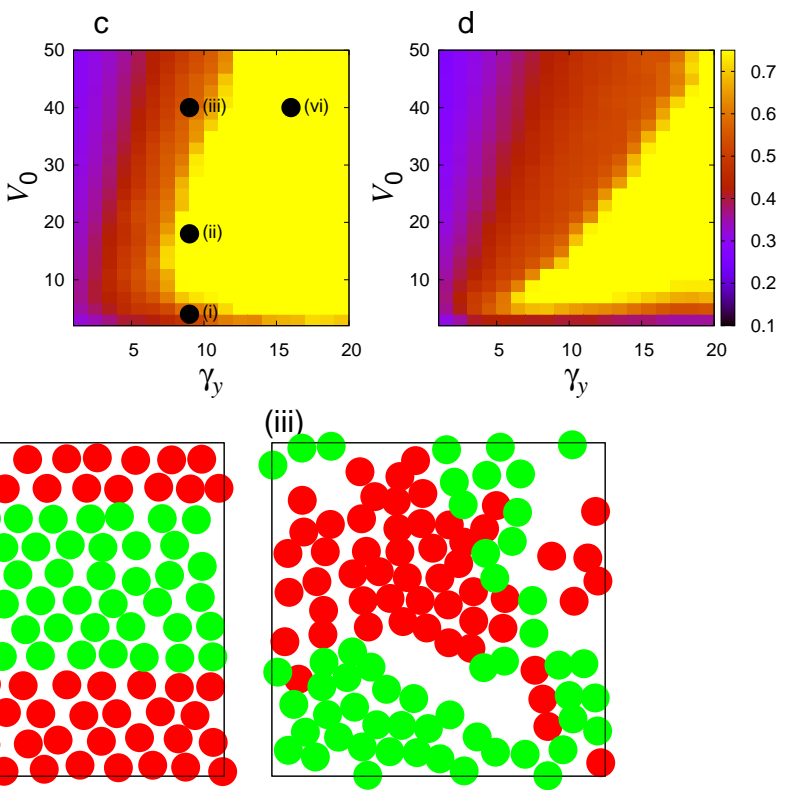

(vi)
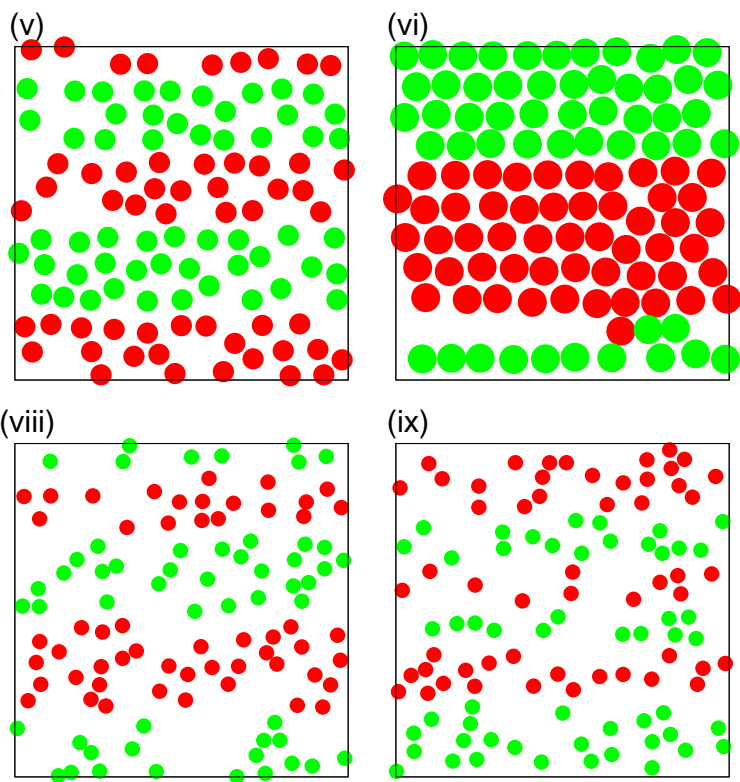

FIG. 1. (Color online) Phase diagrams representing the lane formation order parameter $\Psi$ for various combinations of the friction along the $y$-direction $\gamma_{y}$ and the desired velocity $V_{0}$ at the densities $\rho=0.2$ (a), 0.5 (b), 0.8 (c), and 1.0 (d). Typical snapshots of the states (i)-(ix) in the phase diagrams are also illustrated. In these snapshots, note that the linear dimension of the system is normalized so that the particle size changes with the density.

ation in the $x$-direction is calculated from

$$
\Delta=\frac{1}{2}\left(\left\langle\Delta^{+}\right\rangle+\left\langle\Delta^{-}\right\rangle\right)
$$

where $\Delta^{ \pm}=(2 / N) \sum_{i=1}^{N / 2}\left(v_{i x}^{ \pm}-\left\langle\bar{v}^{ \pm}\right\rangle\right)^{2}$ is the velocity variance in each driven direction.

The number of lanes $N_{\mathrm{L}}$ was also evaluated. For this, the order parameter of each strip $\psi_{k}$ was first discretized with respect to the threshold values \pm 0.5 to $\psi_{k}^{\mathrm{d}}=1\left(\psi_{k}>0.5\right), 0$ $\left(\left|\psi_{k}\right| \leq 0.5\right)$, and $-1\left(\psi_{k}<-0.5\right)$. Then, the nearest $l$-th strip for which the order parameter is $\psi_{l}^{\mathrm{d}} \neq 0$ from the $k$-th lane can be determined as follows:

$$
l(k)=\min _{1<l-k<n_{\mathrm{div}}}\left[l+\left(k+n_{\mathrm{div}}-1\right) \delta_{0, \psi_{l}^{\mathrm{d}}}\right]
$$

Here, note that the maximum and minimum of the index $l$ are given by $k+n_{\mathrm{div}}-1$ and $k+1$, respectively. In addition, the periodic boundary condition $\psi_{j+n_{\text {div }}}^{\mathrm{d}}=\psi_{j}^{\mathrm{d}}$ was utilized. Because the number of lanes $N_{\mathrm{L}}$ is equal to that of the interface between the oppositely driven lanes, the following equation 

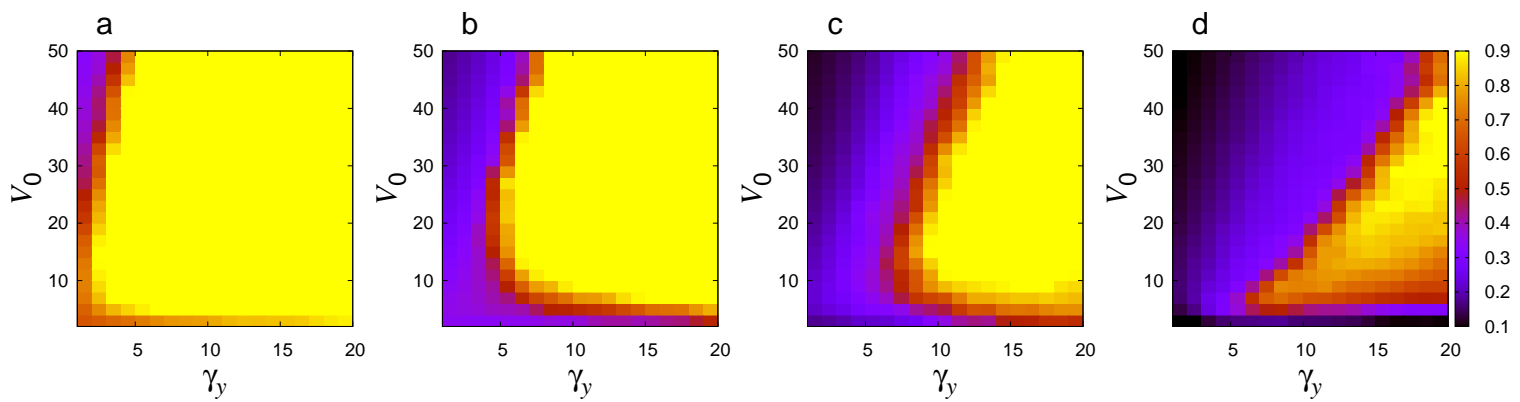

FIG. 2. (Color online) Phase diagrams representing the transport efficiency $\eta$ for various combinations of the friction along the $y$-direction $\gamma_{y}$ and the desired velocity $V_{0}$ at the densities $\rho=0.2$ (a), 0.5 (b), 0.8 (c), and 1.0 (d).
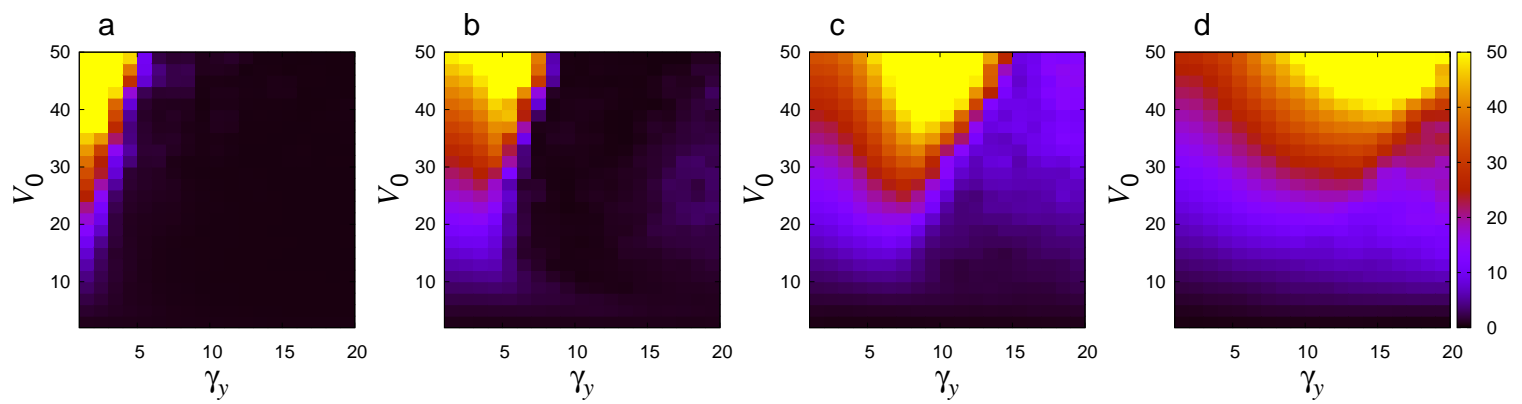

FIG. 3. (Color online) Phase diagrams representing the velocity fluctuation in the $x$-direction $\Delta$ for various combinations of the friction along the $y$-direction $\gamma_{y}$ and the desired velocity $V_{0}$ at the densities $\rho=0.2$ (a), 0.5 (b), 0.8 (c), and 1.0 (d).

can be obtained:

$$
N_{\mathrm{L}}=\left\langle\sum_{k=1}^{n_{\mathrm{div}}} \delta_{-1, \psi_{k}^{\mathrm{d}} \psi_{(k, k)}^{\mathrm{d}}}\right\rangle
$$

The average thickness of the interface $\xi$ was estimated by

$$
\xi=\left\langle\frac{\ell_{\mathrm{div}}}{N_{\mathrm{L}}} \sum_{k=1}^{n_{\mathrm{div}}}(l(k)-k-1) \times \delta_{-1, \psi_{k}^{\mathrm{d}} \psi_{l(k)}^{\mathrm{d}}}\right\rangle,
$$

for $N_{\mathrm{L}} \neq 0$. Correspondingly, the average lane width along the $y$-direction $W_{\mathrm{L}}$ can be evaluated from the following relation:

$$
\frac{L}{N_{\mathrm{L}}}=W_{\mathrm{L}}+\xi
$$

Thus, the average length scale per lane $L / N_{\mathrm{L}}$ can be evaluated from the combination of the lane width $W_{\mathrm{L}}$ and the interface thickness $\xi$.

\section{RESULTS AND DISCUSSION}

\section{A. Density dependence on lane formation at $N=108$}

First, we show the results of various observables in the system with $N=108$. In particular, the density dependence is systematically discussed in this subsection. Figure 1 plots the lane formation order parameters $\Psi$ for various combinations

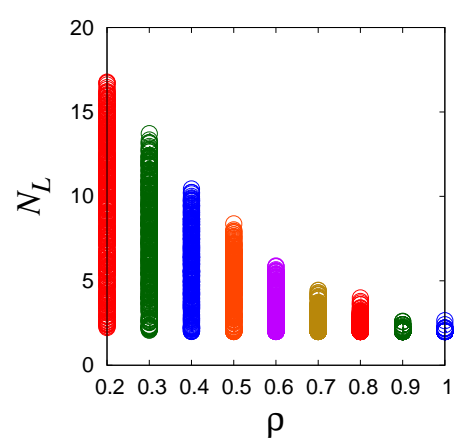

FIG. 4. (Color online) Lane number $N_{\mathrm{L}}$ of the laned states ( $\Psi \geq 0.8$ ) as a function of the density $\rho$.

of $\gamma_{y}$ and $V_{0}$. Snapshots of the states (i)-(ix) in the phase diagrams are also illustrated. We confirm that the transition lines in phase diagrams eventually converged at final stages in our simulations. Thus, the utilized simulation time is sufficient to achieve the steady states. The characteristic time of lane formation will be discussed later. These figures indicate that both large $V_{0}$ and $\gamma_{y}$ led to lane formation. With increasing $V_{0}$, the collision between oppositely driven particles was enhanced. In addition, increasing $\gamma_{y}$ reduced the mobility in the $y$-direction perpendicular to the driven direction. These two effects resulted in a bundle of particles in the same direction and generated the interface between oppositely driven particles, as seen in the snapshots of Fig. 1. This was the main 

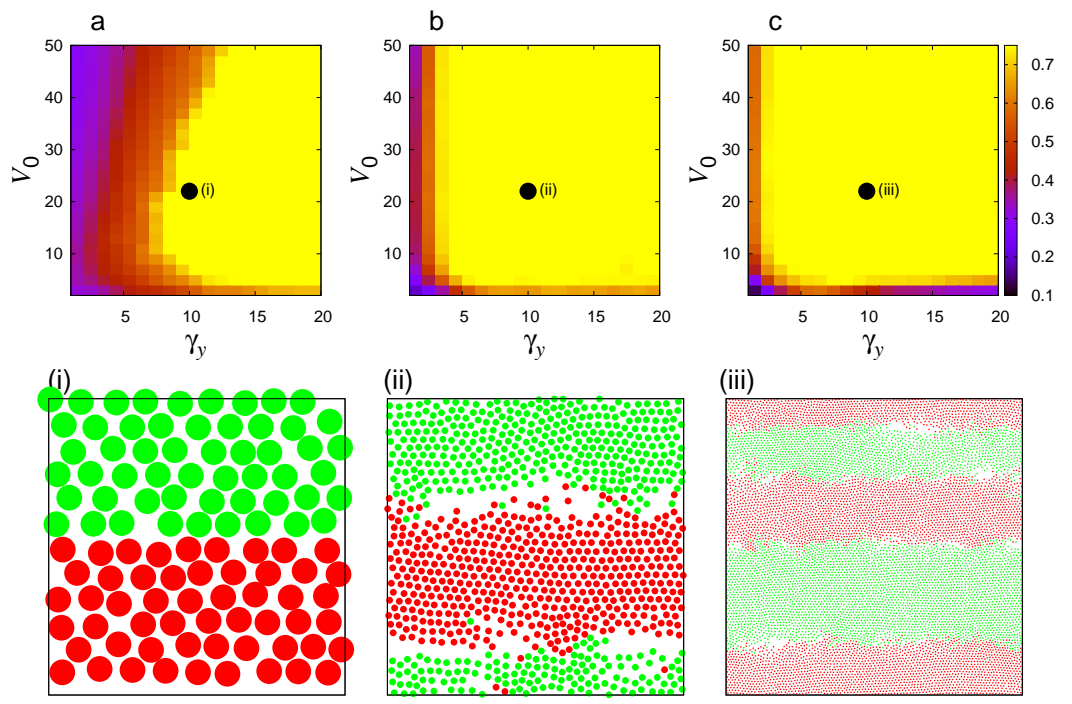

FIG. 5. (Color online) Phase diagrams representing the lane formation order parameter $\Psi$ for various combinations of the friction along the $y$-direction $\gamma_{y}$ and the desired velocity $V_{0}$ at the density $\rho=0.8$ for the system sizes $N=108$ (a), 1000 (b), and 10000 (c). Typical snapshots of the state $\left(\gamma_{y}, V_{0}\right)=(10,22)$ are illustrated as (i)-(iii). In these snapshots, note that the linear dimension of the system is normalized so that the particle size changes with the density.

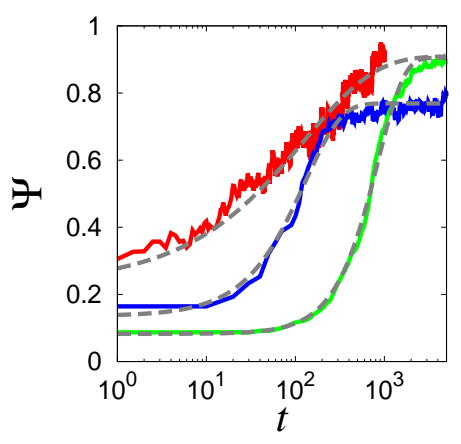

FIG. 6. (Color online) Time development of the lane formation order parameter $\Psi$ with the particle number $N=108$ (red), 1000 (blue), and 10000 (green). The state was set to $\left(\gamma_{y}, V_{0}\right)=(10,22)$, which is the same as the snapshot in Fig. 5 The dashed lines represent the fitting results with Eq. 11.

mechanism for the lane formation, which is in accord with the previous study using Newtonian dynamics [36]. Figure 2 plots a contour map of the transport efficiency $\eta$. This indicates that the overall behaviors of $\Psi$ and $\eta$ were well correlated. Namely, higher lane formation provided more efficient transportation.

Both Figs. 1 and 2 demonstrate that the region of the lane formation states became smaller as the density increased. This can be understood as follows. The mean free path of the particle decreased with increasing density. Accordingly, a position exchange after a collision between oppositely driven particles is unlikely to occur in such dense states. In addition, the transition line between nonlane and lane states showed nonmonotonic and reentrant features, particularly at higher densities. That is, the lane state reentered the no-lane state when $V_{0}$ was greatly increased at a fixed $\gamma_{y}$ [see Figs. [1(i)-(iii)]. To visualize the details of the reentrance, the corresponding velocity fluctuation in the driven $x$-direction $\Delta$ was analyzed, as illustrated in Fig. 3. The no-lane states with larger $V_{0}$ exhibited markedly larger velocity fluctuations at all densities. Figure 1 shows a typical snapshot at $\rho=0.8$ as state (iii). The interface between opposite lanes became unstable, and the same species tended to aggregate to form a cluster. Finally, the lane structure became a clustered structure, where collisions of oppositely driven clusters frequently occurred.

Note that the observed reentrance is not equivalent to similar phenomena observed in the lane formation of externally driven particles [18]. In the latter, the reentrant behavior between the no-lane and lane structures demonstrated dependence on the density at some fixed external force. Specifically, lane formation was unlikely because the thermal fluctuation became dominant at low densities even with a large external force. In contrast, as seen in Figs. 1 and 2 the transition line of the present self-driven system monotonically changed as a function of density. This was due to the anisotropic friction $\gamma_{y}$, which reduced the fluctuation of the mobility in the $y$-direction even at low densities.

The snapshots of states (iv)-(vi) in Fig. 1 show that the number of lanes $N_{\mathrm{L}}$ apparently depended on the density $\rho$. Figure 4 presents a scatter plot of $N_{\mathrm{L}}$ with various $V_{0}$ and $\gamma_{y}$ as a function of the density $\rho$. This indicates that $N_{\mathrm{L}}$ gradually decreased with increasing $\rho$. As mentioned above, lane formation is caused by the collision between oppositely driven particles. At high densities, where the mean free path becomes smaller, the collisions of oppositely driven particles are correlated to one another; then, the aftereffects are plausibly widespread to some extent to make a wider bundle of samespecies particles. This leads to a smaller number of lanes at higher densities. The range of $N_{\mathrm{L}}$ was observed to become 

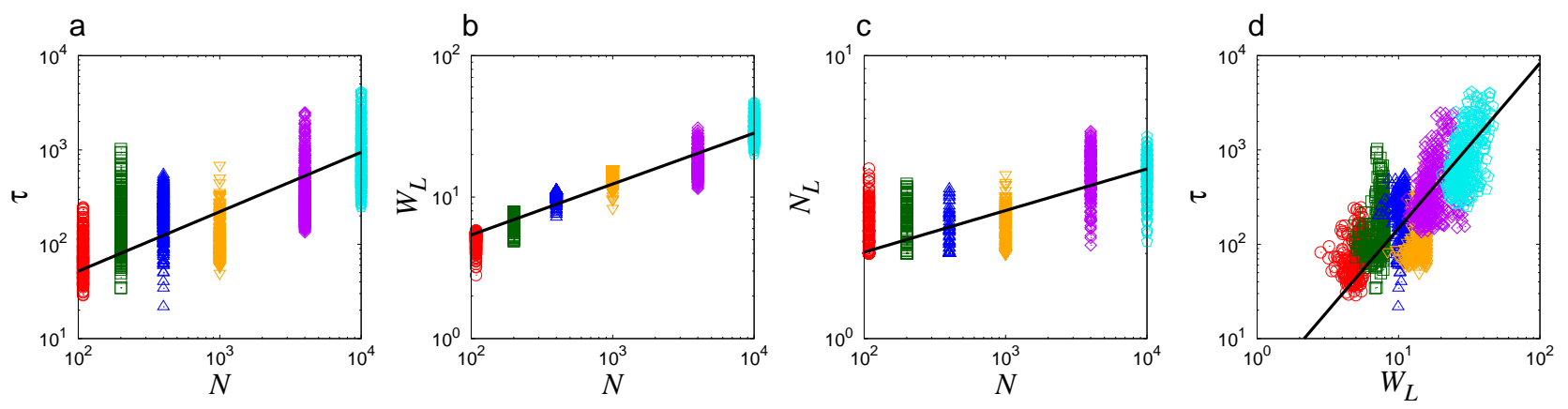

FIG. 7. (Color online) Particle number dependence of the lane formation coarsening time $\tau$ (a), lane width $W_{\mathrm{L}}$ (b), and lane number $N_{\mathrm{L}}$ (c). The states were chosen for $\Psi \geq 0.8$. The straight lines represents the power laws $N^{0.63}$ (a), $N^{0.36}$ (b), and $N^{0.15}$ (c), which were determined by fitting for the plotted data of each figure. (d) Relationship between $\tau$ and $W_{\mathrm{L}}$. The scaling $\tau \sim W_{\mathrm{L}}^{1.75}$ is represented by a straight line.
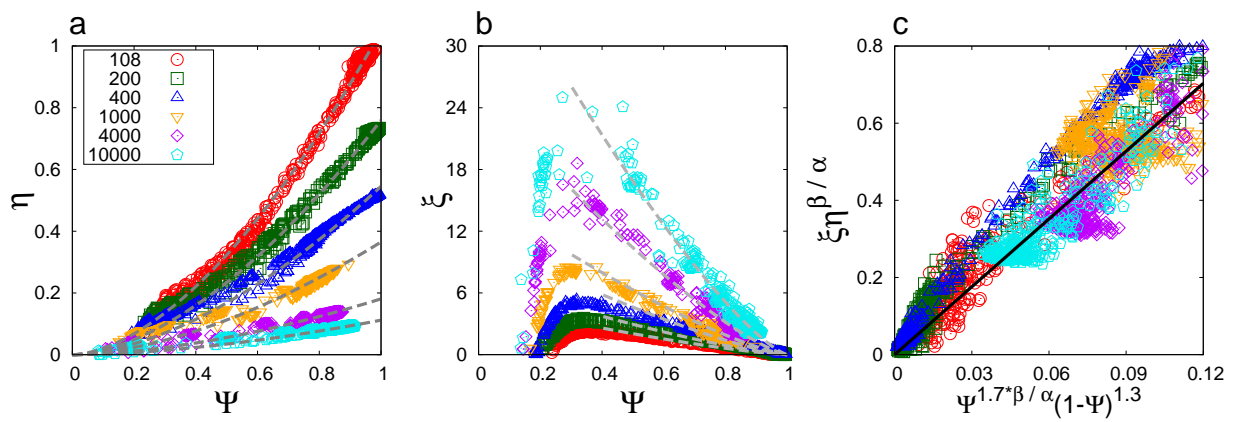

FIG. 8. (a) Transport efficiency $\eta$ as a function of the lane formation order parameter $\Psi$ for various particle numbers $N$. The dashed curves represent the fitting results using the function $\eta=A N^{-\alpha} \Psi^{1.7}$ with the constant $A=10.2$ and the exponent $\alpha=0.49$. (b) Interface thickness $\xi$ as a function of the lane formation order parameter $\Psi$. The dashed curves represent the fitting results using the function $\xi=B N^{\beta}(1-\Psi)^{1.3}$ with the constant $B=0.73$ and the exponent $\beta=0.44$. Fitting was performed for $\Psi \geq 0.4$. (c) Scaling relationship between $\Psi^{1.7 \beta / \alpha}(1-\Psi)^{1.3}$ and $\xi \eta^{\beta / \alpha}$. The states were chosen for $\Psi \geq 0.4$. The straight line represents the proportional relationship with the slope of $A^{\beta / \alpha} B$.

progressively wider when the density decreased. The snapshots of states (vii)-(ix) $\left(\gamma_{y}=16, V_{0}=40\right)$ in Fig. 1 show the strong dependence of $N_{\mathrm{L}}$ on $V_{0}$ with a fixed $\gamma_{y}=5$ at the density $\rho=0.2$. In fact, the fluctuation of the mobility in the $y$-direction became smaller with a larger $\gamma_{y}$. This resulted in a larger $N_{\mathrm{L}}$ because the collisions were less correlated than those at higher densities. In contrast, both larger $V_{0}$ and smaller $\gamma_{y}$ reduced $N_{\mathrm{L}}$ even at low densities, for which the mechanism was similar to that at higher densities. This effect led to the broadly scattered distribution at lower densities, as seen in Fig. 4 An analogous density dependence of the lane number was previously reported for the external-field-driven systems [21]. In the study, however, the particle number was controlled to change the density in the fixed simulation area. This means that the system size effect has not been adequately investigated in a systematic manner. To overcome this issue, we explored the details of the system size effect on lane formation, as described in the next subsection.

\section{B. Finite-size effect on lane formation}

In this subsection, we discuss the finite-size effect on the lane formation of the oppositely self-driven particles. Figure 5 shows the system size dependence of the lane formation order parameter $\Psi$. Apparently, the extent of the capability to form the laned structure increased with the system size $N$. In particular, the clustered structure that was exhibited for larger $V_{0}$ in the $N=108$ system eventually disappeared for larger $N$ systems at any state in the phase diagram. The snapshots in Fig. 5 show that the geometric structures of the lane such as the lane number $N_{\mathrm{L}}$, the lane width $W_{\mathrm{L}}$, and the interface thickness $\xi$, were affected by the system size even when the parameters $\gamma_{y}$ and $V_{0}$ had the same values.

For more details, we examined the coarsening process of the lane formation with the time evolution of $\Psi(t)$. The averaged results of the state at $\gamma_{y}=10$ and $V_{0}=22$ are shown in Fig. 6for $N=108,1000$, and 10000. The characteristic time scale associated with the lane formation from the randomly mixed configuration became slower when the system size increased. This lane formation time scale is quantified by the following function:

$$
\Psi(t)=\Psi_{\text {in }}+\left(\Psi_{\text {st }}-\Psi_{\text {in }}\right)\left[1-e^{-(t / \tau)^{c}}\right],
$$

where $\Psi_{\text {in }}$ and $\Psi_{\text {st }}$ are given by the lane formation order parameter at the initial and steady states, respectively. Thus, the fitting parameters are the exponent $c$ and the characteristic time $\tau$. The results are plotted in Fig. 6 Figure 7 (a) plots the quantified time scale $\tau$ as a function of $N$ for the 
state $\Psi \geq 0.8$. The characteristic time of the coarsening $\tau$ scaled as $\tau \propto N^{0.63}\left(\propto L^{1.26}\right)$ on average. This implies that the macroscopic lane formation occurs at the thermodynamic limit. A comparable system size dependence has been reported for the lane formation caused by the external field [25]. Furthermore, analogous scaling has been demonstrated in the coarsening dynamics of the driven lattice-gas model [37-40]. In addition, driven on- and off-lattice model simulations have recently been compared [30].

The system size dependences of the lane width $W_{\mathrm{L}}$ and the lane number $N_{\mathrm{L}}$ are plotted in Figs.7(b) and (c), respectively. If the system size effect is negligible, the lane width $W_{\mathrm{L}}$ should converge to some finite value; then, $N_{\mathrm{L}}$ increases according to $N_{\mathrm{L}} \propto L(\propto \sqrt{N})$. However, as mentioned above, the lane formation can only exist with infinite time. Correspondingly, the lane width approximately followed $W_{\mathrm{L}} \propto N^{0.36}\left(\propto L^{0.72}\right)$ on average, which is weaker than the proportional relation to the system linear dimension $L$. Indeed, Fig. 7(c) indicates that the lane number $N_{\mathrm{L}}$ slightly increased with the particle number $N$. In other words, the two-lane state with the width $L / 2$ could not develop in the finite time simulations. The relationship between the length and time scales $\tau \sim W_{\mathrm{L}}{ }^{1.75}$ was eventually obtained, as shown in Fig. 7(d). These numerical results can be understood as the dynamical effect of the collisions of opposite particles. The two-lane state is energetically the most stable because the interface effect of the opposite driven particles is lowest. However, when a lane with the width $W_{\mathrm{L}}=L / 2$ is formed from the disordered initial configuration, the number of collisions between different species increases with the system size. To reduce such energy loss due to collisions during the coarsening process, the lane should be split to increase the lane width $W_{\mathrm{L}} \propto L^{0.72}$, as shown in Fig. 7 (b).

Finally, the system size effect of the transport efficiency $\eta$ was examined. Figure 8 (a) plots the relationship between $\Psi$ and $\eta$ for various particle numbers $N$. As discussed in Sect. IVA the lane formation order parameter $\Psi$ correlated with $\eta$ at $N=108$. However, the overall efficiency $\eta$ decreased even at the larger $\Psi$ state for $N=10000$. This dependence of $\eta$ on $\Psi$ can be described by the proportional relation as $\eta=A N^{-\alpha} \Psi^{1.7}$ with the constant $A \simeq 10.2$ and the exponent $\alpha \simeq 0.49$. As noted above, particles of different species within the interface collided frequently, which reduced the transport efficiency $\eta$. Indeed, the number of the interface slightly increased with $N_{\mathrm{L}}$, as shown in Fig. 7 (c). However, the observed reduction of $\eta$ with increasing $N$ was rather substantial. To clarify this phenomenon, the interface thickness $\xi$ was plotted as a function of the degree of the lane formation $\Psi$, as shown in Fig. 8(b). The interface thickness $\xi$ increased with a higher particle number $N$. According to the definition given in Eq. [10], $\xi$ becomes zero at the nonlane state $\Psi=0$ with $N_{\mathrm{L}}=0$. As shown in the figure, the peak was remarkably pronounced at the intermediate ordered state $\Psi \simeq 0.3$. Furthermore, $\xi$ strongly decreased toward zero when $\Psi$ approached unity. This reduction behavior can be described by $\xi=B N^{\beta}(1-\Psi)^{1.3}$ with the constant $B \simeq 0.73$ and the exponent $\beta \simeq 0.44$. From the two equations, the particle number $N$ dependence can be deleted; thus, the relationship among the three variables $\Psi, \xi$, and $\eta$ is given by $\Psi^{1.7 \alpha / \beta}(1-\Psi)^{1.3} \sim \xi \eta^{\alpha / \beta}$, as presented in Fig. 8 (c).

\section{SUMMARY}

We numerically studied the lane formation dynamics of oppositely self-driven particles based on the social force model. In particular, we addressed the density and finite-size effects on the lane formation process.

First, the density dependence of the lane formation order parameter $\Psi$ was analyzed. The transition lines that distinguish the no-lane and lane states can be described by various combinations of the desired velocity $V_{0}$ and the anisotropic friction coefficient $\gamma_{y}$. The density dependence of the transition line monotonically changes, i.e., the area of the lane formation region decreases with increasing density. Such monotonic behavior is contrary to the observed lane formation caused by an external field, where the reentrant effect is exhibited in the density dependence of the critical external force for lane formation [18]. This discrepancy is associated with the friction $-\gamma_{y} v_{y}$, which reduces the fluctuation in the direction perpendicular to the driven direction. Thus, the ability of the lane formation in the present model becomes higher than that in Ref.18. Alternatively, we found a different type of reentrant structure in the dynamical phase diagram, particularly at the higher density state. If the desired velocity $V_{0}$ becomes excessively large, the interface of different lanes becomes unstable and forms clustered structures of same-species particles.

Second, the finite-size effect on the lane formation was systematically analyzed. In particular, the system size dependence of the spatiotemporal scales of the lane formation was characterized. The coarsening process of the lane is analogous to the driven lattice-gas model, where the macroscopic twolane state is realized at the thermodynamic limit. By contrast, with finite simulation times, the dynamic effects due to collisions of oppositely driven particles, particularly within the interfaces, cause the lane to split. The system size dependence of the transport efficiency was quantified with two variables: the lane formation order parameter and the interface thickness of different lanes. The obtained scaling relation allows us to predict how efficiently particles flow toward a desired direction by knowing the degree of lanes and interface thickness for any system size.

\section{ACKNOWLEDGMENTS}

The authors thank H. Hayakawa and H. Wada for helpful discussions. K.I. is grateful to Y. Ōno and N. Matubayasi for supporting his stay at Osaka University. This work was partially supported by JSPS KAKENHI Grant Numbers JP26400428 on Scientific Research (c) and JP16H00829 on Innovative Area (2503) Studying the Function of Soft Molecular Systems by the Concerted Use of Theory and Experiment. The computations were performed at Research Center of Computational Science, Okazaki Research Facilities, National Institutes of Natural Sciences, Japan. 
[1] T. Vicsek and A. Zafeiris, Phys. Rep. 517, 71 (2012).

[2] M. C. Marchetti, J. F. Joanny, S. Ramaswamy, T. B. Liverpool, J. Prost, M. Rao, and R. A. Simha, Rev. Mod. Phys. 85, 1143 (2013).

[3] A. M. Menzel, Phys. Rep. 554, 1 (2015).

[4] C. Bechinger, R. Di Leonardo, H. L owen, C. Reichhardt, G. Volpe, and G. Volpe, Rev. Mod. Phys. 88, 045006 (2016).

[5] D. Helbing and T. Vicsek, New J. Phys. 1, 13 (1999).

[6] D. Helbing, Rev. Mod. Phys. 73, 1067 (2001).

[7] C. Castellano, S. Fortunato, and V. Loreto, Rev. Mod. Phys. 81, 591 (2009).

[8] D. Chowdhury, A. Schadschneider, and K. Nishinari, Phys. Life Rev. 2, 318 (2005).

[9] I. Karamouzas, B. Skinner, and S. J. Guy, Phys. Rev. Lett. 113, 238701 (2014).

[10] D. Helbing and P. Molnár, Phys. Rev. E 51, 4282 (1995).

[11] D. Helbing, I. Farkas, and T. Vicsek, Phys. Rev. Lett. 84, 1240 (2000).

[12] D. Helbing, I. Farkas, and T. Vicsek, Nature 407, 487 (2000).

[13] W. J. Yu, R. Chen, L. Y. Dong, and S. Q. Dai, Phys. Rev. E 72, 026112 (2005).

[14] W. Yu and A. Johansson, Phys. Rev. E 76, 046105 (2007).

[15] M. Moussaï, N. Perozo, S. Garnier, D. Helbing, and G. Theraulaz, PLoS ONE 5, e10047 (2010).

[16] F. Zanlungo, T. Ikeda, and T. Kanda, Phys. Rev. E 89, 012811 (2014).

[17] J. Dzubiella, G. Hoffmann, and H. Löwen, Phys. Rev. E 65, 021402 (2002).

[18] J. Chakrabarti, J. Dzubiella, and H. Löwen, Phys. Rev. E 70, 012401 (2004).

[19] M. Rex and H. Löwen, Phys. Rev. E 75, 051402 (2007).

[20] M. Rex and H. Löwen, Eur. Phys. J. E 26, 143 (2008).

[21] X. Liu, W. Ge, and J. Li, Powder Technol. 184, 224 (2008).

[22] A. Wysocki and H. Lowen, J. Phys.: Condens. Matter 23, 284117 (2011).

[23] K. Jiang, C. R. Du, K. R. Sütterlin, A. V. Ivlev, and G. E. Morfill, EPL 92, 65002 (2011).
[24] M. Kohl, A. V. Ivlev, P. Brandt, G. E. Morfill, and H. Lowen, J. Phys.: Condens. Matter 24, 464115 (2012).

[25] T. Glanz and H. Löwen, J. Phys.: Condens. Matter 24, 464114 (2012).

[26] K. Lichtner and S. H. L. Klapp, Europhys. Lett. 106, 56004 (2014).

[27] B. Heinze, U. Siems, and P. Nielaba, Phys. Rev. E 92, 012323 (2015).

[28] F. Kogler and S. H. L. Klapp, Europhys. Lett. 110, 10004 (2015).

[29] M. E. Foulaadvand and B. Aghaee, Eur. Phys. J. E 39, 37 (2016).

[30] K. Klymko, P. L. Geissler, and S. Whitelam, Phys. Rev. E 94, 022608 (2016).

[31] C. W. Wächtler, F. Kogler, and S. H. L. Klapp, Phys. Rev. E 94, 052603 (2016).

[32] M. E. Leunissen, C. G. Christova, A.-P. Hynninen, C. P. Royall, A. I. Campbell, A. Imhof, M. Dijkstra, R. van Roij, and A. van Blaaderen, Nature 437, 235 (2005).

[33] K. Sütterlin, A. Wysocki, A. Ivlev, C. Räth, H. Thomas, M. Rubin-Zuzic, W. Goedheer, V. Fortov, A. Lipaev, V. Molotkov, O. Petrov, G. Morfill, and H. Löwen, Phys. Rev. Lett. 102, 085003 (2009).

[34] T. Vissers, A. Wysocki, M. Rex, H. Lowen, C. P. Royall, A. Imhof, and A. van Blaaderen, Soft Matter 7, 2352 (2011).

[35] T. Vissers, A. van Blaaderen, and A. Imhof, Phys. Rev. Lett. 106, 228303 (2011).

[36] M. Ikeda, H. Wada, and H. Hayakawa, EPL 99, 68005 (2012).

[37] E. Levine, Y. Kafri, and D. Mukamel, Phys. Rev. E 64, 026105 (2001).

[38] P. I. Hurtado, J. Marro, P. L. Garrido, and E. V. Albano, Phys. Rev. B 67, 014206 (2003).

[39] G. P. Saracco and E. V. Albano, J. Chem. Phys. 118, 4157 (2003).

[40] H. Ohta, EPL 99, 40006 (2012). 